\title{
Donovanosis Treated with Thiamphenicol
}

\author{
Walter Belda Junior ${ }^{1}$, Paulo Eduardo Neves Ferreira Velho ${ }^{2}$, Marcelo Arnone ${ }^{3}$ and Ricardo Romitti ${ }^{4}$ \\ ${ }^{1}$ Department of Dermatology, School of Medicine, University of São Paulo; ${ }^{2}$ Department of Internal Medicine (Dermatology), Medical School, \\ State University of Campinas; Campinas, SP; ${ }^{3}$ Department of Dermatology, School of Medicine, University of São Paulo; ${ }^{4}$ Department of \\ Dermatology, School of Medicine, University of São Paulo; São Paulo, SP, Brazil
}

Ten patients with donovanosis were treated with thiamphenicol for two weeks. In eight of them, included two HIV infected patients, lesions healed. The safety profile of thiamphenicol makes it a useful and cost-effective agent in the management of donovanosis. Randomized controlled trials should be conducted with these treatment options. Key-Words: Azithromycin, granuloma Inguinale, sexually transmitted disease, therapy, thiamphenicol.

Donovanosis is an indolent, progressive, ulcerative bacterial disease caused by the intracellular Gram-negative bacterium Calymmatobacterium granulomatis. It mainly affects the skin and subcutaneous tissue of the genital and perianal areas [1]. Although donovanosis is an intrinsically benign condition effectively treated with numerous antibiotics, the disease is associated with substantial morbidity and mortality. Patients often delay seeking treatment for many months, and substantial tissue destruction, including complete erosion of the genitals, may be present [2]. The disease occurs rarely in the United States, although it is endemic in certain tropical and developing areas, including India, Central Australia and southern Africa, Zambia, Morocco and China [1,3]. Recent accurate data for most endemic areas are limited. For its treatment, there is a dilemma regarding recommendation of the optimal schedule for donovanosis. WHO guidelines recommend azithromycin $1 \mathrm{~g}$ immediately, followed by $500 \mathrm{mg}$ daily but does not state the duration of therapy [4]. In Australia, doses of either $1 \mathrm{~g}$ weekly for 4-6 weeks or less if healing is complete, or $500 \mathrm{mg}$ in a single daily dose for one week only were adequate [5]. The Centers for Disease Control and Prevention (CDC) recommend $1 \mathrm{~g}$ weekly for at least three weeks or until all lesions have healed [6]. Thiamphenicol was reported as an alternative to donovanosis treatment [7]. Other antibiotics introduced more recently and known to be effective include: ceftriaxone, norfloxacin and trofloxacin [3].

We describe ten patients with donovanosis that were treated with thiamphenicol. Their inclusion were sequential, based upon that they are 18-year-old or more, no pregnant women, and without concomitant syphilis, gonorrhea, chancroid, or herpes simplex.

\section{Cases}

Ten patients with donovanosis of the penis, attending the Sexually Transmitted Diseases Clinic at the Teaching Hospital of the University of São Paulo in the period between 2002 and 2005, were submitted to treatment with thiamphenicol $2.5 \mathrm{~g}$

Received on 20 February 2007; revised 5 July 2007.

Address for correspondence: Dr. Paulo Eduardo Neves Ferreira Velho. Cidade Universitária "Zeferino Vaz", s/n. Barão Geraldo, Campinas, São Paulo. Zip code: 13083-970. Phone/Fax: 55-19-3289-4107. Email:pvelho@unicamp.br.

The Brazilian Journal of Infectious Diseases 2007;11(4):388-389. (C) 2007 by The Brazilian Journal of Infectious Diseases and Contexto Publishing. All rights reserved. orally on the first day and $1 \mathrm{~g}$ daily during two weeks. The diagnoses were confirmed by smears obtained directly from the tissue and stained with Giemsa, which demonstrated typical intracellular Donovan bodies within large mononuclear cells. The histological examination showed epithelial proliferation with heavy inflammatory infiltrate of plasma cells and some neutrophils, and silver stains revealed sparse Donovan bodies. None of the patients had positive serology indicating active Treponema pallidum infection. Genital infections due to Neisseria gonorrhoeae, Haemophilus ducreyi and herpes simplex virus were excluded by polymerase chain reaction testing. Only two patients had concomitant infection with human immunodeficiency virus 1 (HIV-1).

At the first follow-up examination, seven days after the therapy was started, all patients showed partial regression of the ulcer. No side effects were identified and in all patients the smear obtained remained positive to Donovan bodies.

At the second follow-up evaluation (15 days after the beginning of therapy), in eight cases the ulcers were completely healed, including two patients with HIV-1. The two other cases continued to present genital ulcers, and the smears observed in touch preparation stained with Giemsa's stain were still positive to Donovan bodies. These patients were immediately treated with azithromycin $1 \mathrm{~g}$ and $500 \mathrm{mg}$ daily for two more weeks.

No abnormalities were observed in laboratory exams during or three months after the end of treatment. Just one patient felt mild reactions (headache and nausea). All patients were evaluated 30, 60 and 90 days after the end of therapy and all were still healed.

\section{Discussion}

Donovanosis is an important cause of chronic ulceration and a potentially important risk factor for HIV transmission. In the absence of randomized controlled trials, donovanosis antibiotic treatment is based on the results of clinical experience. There have been conflicting reports on the efficacy of several regimens, and treatment failures have been report with all courses of therapy $[2,3]$. One of the options is treatment with azithromycin, currently accepted as the best drug available for donovanosis [3].

In eight of our ten patients treated with thiamphenicol the lesions were completely resolved in two weeks. Among them were two patients with HIV-1. The two other patients had been treated with azithromycin $1 \mathrm{~g}$ orally immediately then 
$500 \mathrm{mg}$ daily for only two weeks. All patients were followed up until there was no sign of the disease and no recurrence for at least 90 days.

In Brazil, the cost of thiamphenicol treatment was about $40 \%$ of that recommended by CDC guidelines. The two patients that needed to use azithromycin after initial use of thiamphenicol spent just about $10 \%$ more than would be spent if they had used azithromycin for three weeks [8].

The safety profile of thiamphenicol makes it a useful and cost-effective agent in the management of donovanosis. Randomized controlled trials should be conducted with these treatment options.

\section{References}

1. Lupi O., Madkan V., Tyring S.K. Tropical dermatology: bacterial tropical diseases. J Am Acad Dermatol 2006;54:559-78.

2. Hart G. Donovanosis. Clin Infect Dis 1997;25:24-32.

3. O'Farrel N. Donovanosis. Sex Transm Infect 2002;78:452-7.

4. WHO. Guidelines for the management of sexually transmitted infections. 2001.

5. Bowden F.J., Savage J. Azithromycin for the treatment of donovanosis. Sex Transm Infect 1998;74:78-9.

6. CDC. Sexually transmitted diseases treatment guidelines. MMWR 2002;51:RR6.

7. Jardim M.L. Tratamento da donovanose com tianfenicol. An Bras Dermatol 1990;65:93-4.

8. Associação Brasileira de Comércio Farmacêutico. Lista de Preços Atualizada. ABCFarma 2006;178:13-224. 